\title{
European admixture in Chinchorro DNA
}

\author{
Robert Smith
}

\section{Abstract}

It is widely held that, except for migrations from Beringia or Siberia, there was no contact between the Old World and the New World prior to the colonization of North America by the Norse in the late $10^{\text {th }}$ century AD. Analyses of 23 ancient American DNA samples reveal, however, the presence of European admixture in a sample taken from a Chinchorro mummy of northern Chile dated to 3972-3806 BC. This discovery implies a more complex history of the peopling of the Americas than previously accepted.

\section{Introduction}

Mainstream accounts of the peopling of the Americas stipulate that there was a large migration of people from Beringia during the late Pleistocene, and that modern Amerindians derive most of their ancestry from these migrants. It is commonly believed that these migrants were the originators of the Clovis material culture, and it was long asserted that the appearance of these migrants south of Beringia did not predate the earliest datings of Clovis artifacts, around 13,200 years ago. The accumulation of evidence for earlier American settlements has forced mainstream scholars to abandon the latter position, however. Mainstream accounts also acknowledge two later migrations from Siberia, responsible for bringing speakers of Na-Dene and Eskimo-Aleut languages to the Americas.

A small minority of researchers have advanced theories of other migrations to the Americas. Dennis Stanford and Bruce Bradley, observing the many similarities between Clovis technology and that of the Upper Paleolithic Solutrean culture of Western Europe, have theorized that the ancestors of the Clovis people came not from Siberia, but from Europe, by traversing the Atlantic Ocean during the Last Glacial Maximum [1, 2]. In his 1947 crossing of the Pacific on the balsa raft Kon-Tiki [3], and his 1970 crossing of the Atlantic on the reed boat Ra II [4], Thor Heyerdahl demonstrated the feasibility of early transoceanic contacts, and in his written works he produced an abundance of historical, archeological, and anthropological evidence that such contacts had indeed taken place $[5,6,7]$.

Mainstream scholars have continually rejected these other proposed migrations to the Americas, in spite of the evidence from a multitude of scientific disciplines in support of them. Ancient DNA evidence, however, can prove incontrovertibly that these other migrations took place, and such evidence is presented here for the first time. 


\section{Methods}

\section{Data}

The 23 ancient American genomes analyzed are those published in [8]. Genotype calls generated from aligned reads were intersected with a set of 110,817 transversion SNPs (set 1) for principal component analysis, and a set of 228,841 transversion SNPs (set 2) for qpAdm and ADMIXTURE analyses. The resultant numbers of SNPs for each of the ancient American samples are shown in Table 1.

\section{qpAdm analysis}

The program qpAdm [9] was used to estimate mixture coefficients for the ancient American samples, using the following populations:

- Target population: One of the 23 ancient American samples.

- Source population 1: A collection of populations shown to be purely Amerindian in admixture analyses: Mixe, Piapoco, Wichi, Chané, Karitiana, and Surui.

- Source population 2: One of 11 ancient or modern European, Middle Eastern, or South Asian populations.

- $\quad$ Outgroup populations: Mbuti Pygmies, Han Chinese, Nganasans, Eskimos, and Papuans.

\section{Principal component analysis}

Principal component analysis of the ancient American samples was performed using the smartpca program of EIGENSOFT [10], using default parameters and the lsqproject: YES and numoutlieriter: 0 options.

\section{ADMIXTURE analysis}

Model-based clustering analysis of the ancient American samples was performed with the ADMIXTURE program [11], with the number of assumed ancestral populations ranging from $K$ $=4$ to $K=13$.

\section{Results}

\section{qpAdm analysis}

The results of the of the 253 qpAdm analyses are shown in Table 2, which gives the mean source population 2 mixture coefficient, and the coefficients plus or minus one standard error, for each analysis. The analyses are listed in decreasing order of the mixture coefficient lower bounds. 


\section{Principal component analysis}

A plot of the results of a principal component analysis of ancient and modern American, European, and Middle Eastern samples is shown in Figure 1. Figure 2 is a plot of the results of a second principal component analysis, in which the Middle Eastern samples were excluded, leaving only the American and European samples.

\section{ADMIXTURE analysis}

Plots of the results of $K=4,5,6,7,9$, and 13 ADMIXTURE analyses of the ancient American samples, along with other ancient and modern samples, are shown in Figures 3 through 8.

\section{Discussion}

\section{qpAdm analysis}

In Table 2, the 11 analyses in which the Chinchorro mummy sample was the target population have the 11 largest European, Middle Eastern, or South Asian mixture coefficient lower bounds. The mean coefficients for the Chinchorro sample tend to be around 0.45 , with the lower bounds mostly around 0.30 .

\section{Principal component analysis}

In Figure 1, some of the Mayan, Bolivian, and Quechua samples, which ADMIXTURE analyses show to contain up to $17 \%$ European admixture, are shifted to the right of the more pure Amerindian samples, toward the European and Middle Eastern samples. To the right of them is a Mixtec sample which is 23.5\% European, and to the right of that Mixtec sample is the 30-40\% European Chinchorro mummy sample, and also the Pericú sample BC23, which ADMIXTURE analyses also show to have a significant amount of European admixture. Note that in Figure 1 the Chinchorro mummy sample has a positive value of the second principal component, making it shifted in the direction of Europeans rather than Middle Easterners. In Figure 2 the positions of the Amerindian samples are similar, but BC23 is horizontally between the Mixtec sample and the Chinchorro mummy sample.

\section{ADMIXTURE analysis}

In Figures 3 through 8, for all of the different values of $K$, the Chinchorro mummy sample consistently shows between $30 \%$ and $40 \%$ non-Amerindian admixture, and the only other samples that show a pattern of non-Amerindian components similar to that seen in the Chinchorro sample are the European samples from before the Last Glacial Maximum (LGM):

- $\quad K=4$ : The Chinchorro sample is $31.66 \%$ non-Amerindian. It has a large amount of the blue component, and a small amount of the yellow component. The pre-LGM European 
samples also have large amounts of the blue component, and smaller amounts of the yellow component.

- $\quad K=5$ : The Chinchorro sample is 32.13\% non-Amerindian. It has a large amount of the blue component, a very small amount of the yellow component, and some of the purple component. The pre-LGM European samples also have large amounts of the blue component, and smaller amounts of the yellow and purple components.

- $\quad K=6$ : The Chinchorro sample is $32.67 \%$ non-Amerindian. It has large amounts of the blue and green components, and small amounts of the yellow and purple components. The pre-LGM European samples also have large amounts of the blue component, and smaller amounts of the yellow and purple components, and some of them, particularly Kostenki 14 and the Gravettian sample Goyet Q53-1 from Belgium, have some of the green component.

- $\quad K=7$ : The Chinchorro sample is $34.30 \%$ non-Amerindian. It has significant amounts of the plain blue, light blue, and green components, and a small amount of the purple component. The pre-LGM European samples also have significant amounts of the plain blue, light blue, green, and purple components.

- $\quad K=9$ : The Chinchorro sample is $37.00 \%$ non-Amerindian. It has significant amounts of the plain blue, light blue, and plain green components. The pre-LGM European samples also have significant amounts of the plain blue, light blue, and plain green components. The pine green component is completely absent in the Chinchorro sample, and, with the exception of Kostenki 14, it is also completely absent in the pre-LGM European samples. The plain green component is absent in post-Magdalenian Europeans, and the pine green component is present in significant amounts in Europeans from the Copper Age on, which eliminates modern contamination as a possible source of the European admixture in the Chinchorro sample.

- $\quad K=13$ : The Chinchorro sample is $36.49 \%$ non-Amerindian. The Chinchorro sample has significant amounts of the dark blue, light blue, and plain green components, and a small amount of the plain yellow component. The pre-LGM European samples also have significant amounts of the dark blue, light blue, and plain green components, and small amounts of the plain yellow component. The medium blue component is completely absent in the Chinchorro sample, and since the Eastern European and Western Siberian hunter-gatherers are made up mostly of that component, they are excluded as sources of the European admixture in the Chinchorro sample.

\section{Conclusions}

The above qpAdm, principal component, and ADMIXTURE analyses of 23 ancient American 
samples reveal that one of them, taken from a Chinchorro mummy of northern Chile dated to 3972-3806 BC, contains 30-40\% European admixture. That the non-Amerindian admixture present in the Chinchorro sample is more closely related to Europeans than to Middle Easterners is demonstrated by both the principal component and ADMIXTURE analyses. The ADMIXTURE analyses further shed light on exactly which European population the nonAmerindian admixture in the Chinchorro sample might be from: only the pre-LGM Europeans show a pattern of non-Amerindian components similar to that seen in the Chinchorro sample, which strongly suggests that the pre-LGM Aurignacians or Gravettians, or possibly the LGM Solutreans, were the source of the admixture. The Solutreans seem like a particularly likely source population, in light of the ample archeological evidence for their presence in the Americas. A complicating factor in identifying the exact source of the European admixture in the Chinchorro sample is the amount of divergent genetic drift that would have occurred between the arrival of the European source population in the Americas and the time of the Chinchorro individual analyzed. If the Solutreans were the source, and if they arrived in the Americas around 26,000 years ago, then around 20,000 years of divergent genetic drift would have accumulated in their American descendants by the time of the Chinchorro individual. This drift might account for the differences in the exact proportions of the non-Amerindian components in the Chinchorro and pre-LGM European samples, but it could also conceivably result in a somewhat later European population, such as the Magdalenians, not being correctly identified as the true source population. Regardless of the exact source of the European admixture in the Chinchorro sample, the fact that such admixture exists is the first ancient DNA proof of pre-Norse transatlantic contact.

\section{References}

1. Bradley, B. and Stanford, D. 2004. The North Atlantic ice-edge corridor: a possible Paleolithic route to the New World. World Archaeology, 36: 459-78.

2. Stanford, D. and Bradley, B. 2012. Across Atlantic Ice: The Origin of America's Clovis Culture. Berkeley, CA: University of California Press.

3. Heyerdahl, T. 1950. Kon-Tiki: Across the Pacific by Raft. Chicago, IL: Rand McNally.

4. Heyerdahl, T. 1971. The Ra Expeditions. Garden City, NY: Doubleday.

5. Heyerdahl, T. 1952. American Indians in the Pacific: The Theory behind the Kon-Tiki Expedition. London: George Allen \& Unwin.

6. Heyerdahl, T. 1971. Isolationist or Diffusionist? In The Quest for America (ed. Geoffrey Ashe). New York: Praeger Publishers, pp. 115-154.

7. Heyerdahl, T. 1971. The Bearded Gods Speak. In The Quest for America (ed. Geoffrey Ashe). New York: Praeger Publishers, pp. 199-238. 
8. Raghavan, M., et al. 2015. Genomic evidence for the Pleistocene and recent population history of Native Americans. Science, 349: aab3884.

9. Haak W., et al. 2015. Massive migration from the steppe was a source for Indo-European languages in Europe. Nature, 522: 207-211.

10. Patterson N., Price A. L., and Reich D. 2006. Population structure and eigenanalysis. PLoS Genetics, 2: e190.

11. Alexander D. H., Novembre J., and Lange K. 2009. Fast model-based estimation of ancestry in unrelated individuals. Genome Research, 19: 1655-1664. 
Table 1. Description and numbers of SNPs used for 23 ancient American samples.

\begin{tabular}{|c|c|c|c|c|c|}
\hline Sample & Tribe & Region & Age BP & SNP set 1 & SNP set 2 \\
\hline 939 & Unknown & British Columbia & $6260-5890$ & 38,692 & 81,341 \\
\hline MARC1492 & Micmac & New Brunswick & $516-258$ & 15,374 & 27,461 \\
\hline F9 & Unknown & Northwestern Mexico & $>500$ & 684 & 1,320 \\
\hline MOM6 & Unknown & Northwestern Mexico & $>500$ & 1,233 & 2,378 \\
\hline BC23 & Pericú & Baja California Sur & $800-300$ & 1,068 & 2,100 \\
\hline BC25 & Pericú & Baja California Sur & $800-300$ & 21,180 & 38,118 \\
\hline BC27 & Pericú & Baja California Sur & $800-300$ & 1,216 & 2,195 \\
\hline BC28 & Pericú & Baja California Sur & $800-300$ & 1,083 & 2,041 \\
\hline BC29 & Pericú & Baja California Sur & $800-300$ & 5,634 & 10,770 \\
\hline BC30 & Pericú & Baja California Sur & 800-300 & 12,469 & 24,827 \\
\hline Enoque65 & Unknown & Northeastern Brazil & 3635-3483 & 7,433 & 15,098 \\
\hline Chinchorro & Unknown & Northern Chile & $5922-5756$ & 1,067 & 2,055 \\
\hline AM66 & Alacaluf & Chilean Patagonia & 132 & 3,167 & 5,899 \\
\hline AM71 & Alacaluf & Chilean Patagonia & 132 & 13,322 & 24,060 \\
\hline AM72 & Alacaluf & Chilean Patagonia & 132 & 502 & 918 \\
\hline AM73 & Alacaluf & Chilean Patagonia & 132 & 2,398 & 4,290 \\
\hline AM74 & Alacaluf & Chilean Patagonia & 132 & 46,680 & 87,680 \\
\hline MA572 & Selknam & Tierra del Fuego & $\sim 200$ & 1,242 & 2,268 \\
\hline MA575 & Selknam & Tierra del Fuego & $\sim 200$ & 369 & 732 \\
\hline MA577 & Selknam & Tierra del Fuego & $\sim 200$ & 93,774 & 181,308 \\
\hline 890 & Yaghan & Tierra del Fuego & $\sim 200$ & 28,202 & 50,746 \\
\hline 894 & Yaghan & Tierra del Fuego & $\sim 200$ & 66,852 & 121,408 \\
\hline 895 & Yaghan & Tierra del Fuego & $\sim 200$ & 71,415 & 129,719 \\
\hline
\end{tabular}


Table 2. Estimated source population 2 mixture coefficients produced by 253 qpAdm analyses.

\begin{tabular}{|c|c|c|c|c|}
\hline Target population & Source population 2 & $-1 \mathrm{SE}$ & Mean & $+1 \mathrm{SE}$ \\
\hline Chinchorro mummy & Pit Grave CA & 0.3526 & 0.5426 & 0.7326 \\
\hline Chinchorro mummy & Spanish & 0.3141 & 0.4741 & 0.6341 \\
\hline Chinchorro mummy & Iran EN & 0.3070 & 0.4770 & 0.6470 \\
\hline Chinchorro mummy & Mala & 0.2927 & 0.4697 & 0.6467 \\
\hline Chinchorro mummy & NW Asia Minor EN & 0.2868 & 0.4368 & 0.5868 \\
\hline Chinchorro mummy & Pre-LGM Euro & 0.2849 & 0.4749 & 0.6649 \\
\hline Chinchorro mummy & West Euro HG & 0.2841 & 0.4481 & 0.6121 \\
\hline Chinchorro mummy & Georgia HG & 0.2634 & 0.4014 & 0.5394 \\
\hline Chinchorro mummy & Tamil & 0.2595 & 0.4205 & 0.5815 \\
\hline Chinchorro mummy & East Euro HG & 0.2280 & 0.5600 & 0.8920 \\
\hline Chinchorro mummy & Natufian & 0.1447 & 0.3457 & 0.5467 \\
\hline Mex mummy F9 & East Euro HG & 0.1303 & 0.4593 & 0.7883 \\
\hline Mex mummy F9 & Spanish & 0.1033 & 0.2683 & 0.4333 \\
\hline Mex mummy F9 & Pit Grave CA & 0.0964 & 0.2944 & 0.4924 \\
\hline Mex mummy F9 & Tamil & 0.0954 & 0.2844 & 0.4734 \\
\hline Mex mummy F9 & West Euro HG & 0.0924 & 0.3034 & 0.5144 \\
\hline Mex mummy F9 & NW Asia Minor EN & 0.0896 & 0.2476 & 0.4056 \\
\hline Mex mummy F9 & Georgia HG & 0.0880 & 0.2470 & 0.4060 \\
\hline Mex mummy F9 & Iran EN & 0.0720 & 0.2570 & 0.4420 \\
\hline Patagonian AM73 & Natufian & 0.0668 & 0.1698 & 0.2728 \\
\hline Mex mummy F9 & Pre-LGM Euro & 0.0611 & 0.2161 & 0.3711 \\
\hline Micmac MARC1492 & Georgia HG & 0.0359 & 0.0749 & 0.1139 \\
\hline Micmac MARC1492 & Pit Grave CA & 0.0355 & 0.0785 & 0.1215 \\
\hline Micmac MARC1492 & NW Asia Minor EN & 0.0336 & 0.0686 & 0.1036 \\
\hline Brazilian Enoque65 & Natufian & 0.0335 & 0.1025 & 0.1715 \\
\hline Brazilian Enoque65 & Mala & 0.0334 & 0.0874 & 0.1414 \\
\hline Micmac MARC1492 & Spanish & 0.0326 & 0.0706 & 0.1086 \\
\hline Micmac MARC1492 & Iran EN & 0.0323 & 0.0673 & 0.1023 \\
\hline Pericú BC25 & Spanish & 0.0233 & 0.0563 & 0.0893 \\
\hline Brazilian Enoque65 & Tamil & 0.0232 & 0.0792 & 0.1352 \\
\hline Pericú BC25 & West Euro HG & 0.0231 & 0.0591 & 0.0951 \\
\hline
\end{tabular}




\begin{tabular}{|c|c|c|c|c|}
\hline Target population & Source population 2 & $-1 \mathrm{SE}$ & Mean & $+1 \mathrm{SE}$ \\
\hline Pericú BC25 & Georgia HG & 0.0225 & 0.0565 & 0.0905 \\
\hline Pericú BC25 & NW Asia Minor EN & 0.0222 & 0.0532 & 0.0842 \\
\hline Pericú BC25 & Pre-LGM Euro & 0.0220 & 0.0550 & 0.0880 \\
\hline Pericú BC25 & Tamil & 0.0217 & 0.0577 & 0.0937 \\
\hline Micmac MARC1492 & East Euro HG & 0.0209 & 0.0779 & 0.1349 \\
\hline Pericú BC25 & Pit Grave CA & 0.0204 & 0.0604 & 0.1004 \\
\hline Micmac MARC1492 & West Euro HG & 0.0204 & 0.0624 & 0.1044 \\
\hline Pericú BC25 & Mala & 0.0186 & 0.0546 & 0.0906 \\
\hline Pericú BC25 & Iran EN & 0.0184 & 0.0504 & 0.0824 \\
\hline Mex mummy F9 & Mala & 0.0184 & 0.2364 & 0.4544 \\
\hline Micmac MARC1492 & Natufian & 0.0151 & 0.0581 & 0.1011 \\
\hline Micmac MARC1492 & Pre-LGM Euro & 0.0146 & 0.0516 & 0.0886 \\
\hline Brazilian Enoque65 & Georgia HG & 0.0133 & 0.0683 & 0.1233 \\
\hline Pericú BC25 & Natufian & 0.0128 & 0.0498 & 0.0868 \\
\hline Brazilian Enoque65 & Iran EN & 0.0079 & 0.0569 & 0.1059 \\
\hline Brazilian Enoque65 & Pit Grave CA & 0.0061 & 0.0661 & 0.1261 \\
\hline Brazilian Enoque65 & NW Asia Minor EN & 0.0041 & 0.0541 & 0.1041 \\
\hline Mex mummy MOM6 & Natufian & 0.0040 & 0.1150 & 0.2260 \\
\hline Brazilian Enoque65 & Pre-LGM Euro & 0.0036 & 0.0586 & 0.1136 \\
\hline Fuegian 894 & Natufian & 0.0023 & 0.0213 & 0.0403 \\
\hline Brazilian Enoque65 & Spanish & 0.0023 & 0.0553 & 0.1083 \\
\hline Fuegian 890 & Natufian & 0.0022 & 0.0302 & 0.0582 \\
\hline Micmac MARC1492 & Tamil & 0.0014 & 0.0414 & 0.0814 \\
\hline Brazilian Enoque65 & West Euro HG & -0.0004 & 0.0576 & 0.1156 \\
\hline Fuegian MA577 & Georgia HG & -0.0019 & 0.0161 & 0.0341 \\
\hline Fuegian MA577 & West Euro HG & -0.0026 & 0.0174 & 0.0374 \\
\hline Pericú BC25 & East Euro HG & -0.0027 & 0.0493 & 0.1013 \\
\hline Fuegian MA577 & Iran EN & -0.0029 & 0.0141 & 0.0311 \\
\hline Fuegian MA577 & NW Asia Minor EN & -0.0034 & 0.0136 & 0.0306 \\
\hline Fuegian MA577 & Pit Grave CA & -0.0036 & 0.0184 & 0.0404 \\
\hline Fuegian MA577 & Spanish & -0.0042 & 0.0148 & 0.0338 \\
\hline Fuegian MA577 & Tamil & -0.0042 & 0.0158 & 0.0358 \\
\hline
\end{tabular}




\begin{tabular}{|c|c|c|c|c|}
\hline Target population & Source population 2 & $-1 \mathrm{SE}$ & Mean & $+1 \mathrm{SE}$ \\
\hline Patagonian AM66 & Natufian & -0.0045 & 0.0995 & 0.2035 \\
\hline Fuegian MA577 & Pre-LGM Euro & -0.0056 & 0.0124 & 0.0304 \\
\hline Fuegian MA577 & Mala & -0.0074 & 0.0136 & 0.0346 \\
\hline Micmac MARC1492 & Mala & -0.0093 & 0.0327 & 0.0747 \\
\hline Fuegian MA577 & East Euro HG & -0.0097 & 0.0173 & 0.0443 \\
\hline Fuegian MA577 & Natufian & -0.0103 & 0.0087 & 0.0277 \\
\hline Fuegian 890 & East Euro HG & -0.0125 & 0.0305 & 0.0735 \\
\hline Fuegian 894 & NW Asia Minor EN & -0.0127 & 0.0053 & 0.0233 \\
\hline Fuegian 894 & West Euro HG & -0.0138 & 0.0072 & 0.0282 \\
\hline Fuegian 894 & Spanish & -0.0140 & 0.0060 & 0.0260 \\
\hline Fuegian 894 & Iran EN & -0.0141 & 0.0049 & 0.0239 \\
\hline Fuegian 894 & Georgia HG & -0.0144 & 0.0056 & 0.0256 \\
\hline Fuegian 894 & Pit Grave CA & -0.0145 & 0.0085 & 0.0315 \\
\hline Fuegian 894 & Pre-LGM Euro & -0.0153 & 0.0047 & 0.0247 \\
\hline Fuegian 890 & West Euro HG & -0.0173 & 0.0137 & 0.0447 \\
\hline Fuegian 890 & Georgia HG & -0.0175 & 0.0105 & 0.0385 \\
\hline Patagonian AM74 & Natufian & -0.0176 & 0.0064 & 0.0304 \\
\hline Patagonian AM66 & Pre-LGM Euro & -0.0181 & 0.0539 & 0.1259 \\
\hline Pericú BC28 & Pre-LGM Euro & -0.0185 & 0.0945 & 0.2075 \\
\hline Fuegian 890 & Pre-LGM Euro & -0.0189 & 0.0091 & 0.0371 \\
\hline Fuegian 890 & Iran EN & -0.0194 & 0.0076 & 0.0346 \\
\hline Fuegian 894 & East Euro HG & -0.0198 & 0.0102 & 0.0402 \\
\hline Fuegian 890 & Spanish & -0.0199 & 0.0081 & 0.0361 \\
\hline Fuegian 890 & NW Asia Minor EN & -0.0199 & 0.0071 & 0.0341 \\
\hline Fuegian 890 & Mala & -0.0201 & 0.0119 & 0.0439 \\
\hline Fuegian 890 & Pit Grave CA & -0.0202 & 0.0138 & 0.0478 \\
\hline Fuegian 890 & Tamil & -0.0208 & 0.0102 & 0.0412 \\
\hline Patagonian AM66 & West Euro HG & -0.0210 & 0.0540 & 0.1290 \\
\hline Patagonian AM66 & Iran EN & -0.0212 & 0.0448 & 0.1108 \\
\hline Pericú BC23 & Natufian & -0.0218 & 0.2042 & 0.4302 \\
\hline Fuegian 894 & Tamil & -0.0219 & 0.0001 & 0.0221 \\
\hline Patagonian AM66 & NW Asia Minor EN & -0.0224 & 0.0456 & 0.1136 \\
\hline
\end{tabular}




\begin{tabular}{|c|c|c|c|c|}
\hline Target population & Source population 2 & $-1 \mathrm{SE}$ & Mean & $+1 \mathrm{SE}$ \\
\hline Patagonian AM66 & Spanish & -0.0235 & 0.0525 & 0.1285 \\
\hline Fuegian 894 & Mala & -0.0244 & -0.0014 & 0.0216 \\
\hline Patagonian AM74 & NW Asia Minor EN & -0.0246 & -0.0046 & 0.0154 \\
\hline Patagonian AM74 & Iran EN & -0.0247 & -0.0047 & 0.0153 \\
\hline Patagonian AM74 & East Euro HG & -0.0248 & 0.0072 & 0.0392 \\
\hline Patagonian AM74 & Spanish & -0.0253 & -0.0043 & 0.0167 \\
\hline Patagonian AM66 & Georgia HG & -0.0256 & 0.0524 & 0.1304 \\
\hline Patagonian AM74 & Georgia HG & -0.0256 & -0.0046 & 0.0164 \\
\hline Patagonian AM66 & Pit Grave CA & -0.0276 & 0.0634 & 0.1544 \\
\hline Patagonian AM74 & Pit Grave CA & -0.0276 & -0.0026 & 0.0224 \\
\hline Patagonian AM74 & West Euro HG & -0.0280 & -0.0040 & 0.0200 \\
\hline Patagonian AM74 & Pre-LGM Euro & -0.0288 & -0.0068 & 0.0152 \\
\hline Mex mummy MOM6 & Iran EN & -0.0305 & 0.0945 & 0.2195 \\
\hline Patagonian AM66 & Mala & -0.0312 & 0.0488 & 0.1288 \\
\hline Patagonian AM74 & Tamil & -0.0332 & -0.0092 & 0.0148 \\
\hline Pericú BC29 & Iran EN & -0.0340 & 0.0160 & 0.0660 \\
\hline Mex mummy MOM6 & NW Asia Minor EN & -0.0352 & 0.0698 & 0.1748 \\
\hline Patagonian AM74 & Mala & -0.0364 & -0.0124 & 0.0116 \\
\hline Patagonian AM66 & Tamil & -0.0366 & 0.0444 & 0.1254 \\
\hline Pericú BC29 & NW Asia Minor EN & -0.0368 & 0.0172 & 0.0712 \\
\hline Pericú BC29 & Spanish & -0.0383 & 0.0177 & 0.0737 \\
\hline Pericú BC29 & Georgia HG & -0.0396 & 0.0204 & 0.0804 \\
\hline Fuegian 895 & Natufian & -0.0435 & -0.0235 & -0.0035 \\
\hline Pericú BC29 & West Euro HG & -0.0438 & 0.0182 & 0.0802 \\
\hline Pericú BC29 & Tamil & -0.0438 & 0.0172 & 0.0782 \\
\hline Pericú BC28 & Georgia HG & -0.0441 & 0.0779 & 0.1999 \\
\hline Mex mummy MOM6 & Spanish & -0.0447 & 0.0723 & 0.1893 \\
\hline Fuegian 895 & NW Asia Minor EN & -0.0461 & -0.0271 & -0.0081 \\
\hline Pericú BC29 & Pit Grave CA & -0.0468 & 0.0192 & 0.0852 \\
\hline Fuegian 895 & Iran EN & -0.0469 & -0.0279 & -0.0089 \\
\hline Fuegian 895 & Georgia HG & -0.0474 & -0.0284 & -0.0094 \\
\hline Patagonian AM71 & Natufian & -0.0479 & -0.0049 & 0.0381 \\
\hline
\end{tabular}




\begin{tabular}{|c|c|c|c|c|}
\hline Target population & Source population 2 & $-1 \mathrm{SE}$ & Mean & $+1 \mathrm{SE}$ \\
\hline Fuegian 895 & Spanish & -0.0481 & -0.0281 & -0.0081 \\
\hline Fuegian 895 & Pre-LGM Euro & -0.0507 & -0.0307 & -0.0107 \\
\hline Pericú BC28 & Spanish & -0.0507 & 0.0733 & 0.1973 \\
\hline Fuegian 895 & West Euro HG & -0.0517 & -0.0307 & -0.0097 \\
\hline Pericú BC29 & Mala & -0.0520 & 0.0090 & 0.0700 \\
\hline Patagonian AM66 & East Euro HG & -0.0530 & 0.0480 & 0.1490 \\
\hline Brazilian Enoque65 & East Euro HG & -0.0540 & 0.0210 & 0.0960 \\
\hline Pericú BC28 & NW Asia Minor EN & -0.0545 & 0.0615 & 0.1775 \\
\hline Fuegian 895 & Pit Grave CA & -0.0551 & -0.0321 & -0.0091 \\
\hline Pericú BC29 & Pre-LGM Euro & -0.0553 & 0.0027 & 0.0607 \\
\hline Pericú BC28 & West Euro HG & -0.0568 & 0.0822 & 0.2212 \\
\hline Mex mummy F9 & Natufian & -0.0598 & 0.1282 & 0.3162 \\
\hline Fuegian 895 & Tamil & -0.0601 & -0.0381 & -0.0161 \\
\hline Pericú BC28 & Pit Grave CA & -0.0602 & 0.0798 & 0.2198 \\
\hline Mex mummy MOM6 & Georgia HG & -0.0603 & 0.0877 & 0.2357 \\
\hline Pericú BC28 & Mala & -0.0610 & 0.0850 & 0.2310 \\
\hline Pericú BC28 & Iran EN & -0.0626 & 0.0634 & 0.1894 \\
\hline Fuegian 895 & Mala & -0.0647 & -0.0417 & -0.0187 \\
\hline Pericú BC27 & Natufian & -0.0650 & 0.0570 & 0.1790 \\
\hline Fuegian 895 & East Euro HG & -0.0651 & -0.0361 & -0.0071 \\
\hline Pericú BC28 & East Euro HG & -0.0678 & 0.1322 & 0.3322 \\
\hline Pericú BC28 & Tamil & -0.0699 & 0.0641 & 0.1981 \\
\hline Pericú BC30 & NW Asia Minor EN & -0.0710 & -0.0320 & 0.0070 \\
\hline Pericú BC30 & Iran EN & -0.0720 & -0.0330 & 0.0060 \\
\hline Pericú BC30 & Georgia HG & -0.0722 & -0.0312 & 0.0098 \\
\hline Mex mummy MOM6 & Pre-LGM Euro & -0.0730 & 0.0480 & 0.1690 \\
\hline Mex mummy MOM6 & West Euro HG & -0.0736 & 0.0794 & 0.2324 \\
\hline Pericú BC30 & Spanish & -0.0741 & -0.0331 & 0.0079 \\
\hline Pericú BC29 & Natufian & -0.0744 & -0.0044 & 0.0656 \\
\hline Mex mummy MOM6 & Pit Grave CA & -0.0783 & 0.0647 & 0.2077 \\
\hline Pericú BC27 & Georgia HG & -0.0786 & 0.0514 & 0.1814 \\
\hline Pericú BC30 & West Euro HG & -0.0814 & -0.0374 & 0.0066 \\
\hline
\end{tabular}




\begin{tabular}{|c|c|c|c|c|}
\hline Target population & Source population 2 & $-1 \mathrm{SE}$ & Mean & $+1 \mathrm{SE}$ \\
\hline Pericú BC23 & Tamil & -0.0818 & 0.0732 & 0.2282 \\
\hline Pericú BC30 & Pit Grave CA & -0.0831 & -0.0361 & 0.0109 \\
\hline Pericú BC30 & Natufian & -0.0856 & -0.0376 & 0.0104 \\
\hline Patagonian AM72 & Mala & -0.0864 & 0.1236 & 0.3336 \\
\hline Pericú BC27 & NW Asia Minor EN & -0.0871 & 0.0229 & 0.1329 \\
\hline Patagonian AM72 & NW Asia Minor EN & -0.0884 & 0.1086 & 0.3056 \\
\hline Pericú BC30 & Tamil & -0.0900 & -0.0450 & 0.0000 \\
\hline Pericú BC30 & Pre-LGM Euro & -0.0904 & -0.0464 & -0.0024 \\
\hline Pericú BC23 & Georgia HG & -0.0906 & 0.0554 & 0.2014 \\
\hline Pericú BC23 & Iran EN & -0.0919 & 0.0571 & 0.2061 \\
\hline British Columbia 939 & NW Asia Minor EN & -0.0923 & -0.0673 & -0.0423 \\
\hline Pericú BC29 & East Euro HG & -0.0932 & -0.0072 & 0.0788 \\
\hline Pericú BC23 & Spanish & -0.0934 & 0.0546 & 0.2026 \\
\hline Pericú BC23 & Pre-LGM Euro & -0.0934 & 0.0716 & 0.2366 \\
\hline Pericú BC23 & NW Asia Minor EN & -0.0934 & 0.0536 & 0.2006 \\
\hline Pericú BC30 & Mala & -0.0935 & -0.0495 & -0.0055 \\
\hline British Columbia 939 & Iran EN & -0.0936 & -0.0676 & -0.0416 \\
\hline Pericú BC27 & Spanish & -0.0949 & 0.0251 & 0.1451 \\
\hline Patagonian AM71 & Iran EN & -0.0953 & -0.0593 & -0.0233 \\
\hline British Columbia 939 & Georgia HG & -0.0962 & -0.0692 & -0.0422 \\
\hline Mex mummy MOM6 & Tamil & -0.0966 & 0.0454 & 0.1874 \\
\hline Patagonian AM73 & Iran EN & -0.0972 & -0.0262 & 0.0448 \\
\hline British Columbia 939 & Spanish & -0.0978 & -0.0708 & -0.0438 \\
\hline Patagonian AM73 & NW Asia Minor EN & -0.0979 & -0.0249 & 0.0481 \\
\hline Patagonian AM72 & Georgia HG & -0.0984 & 0.1506 & 0.3996 \\
\hline Patagonian AM72 & Iran EN & -0.0988 & 0.0842 & 0.2672 \\
\hline Patagonian AM72 & Spanish & -0.1007 & 0.1043 & 0.3093 \\
\hline Patagonian AM71 & NW Asia Minor EN & -0.1014 & -0.0644 & -0.0274 \\
\hline Patagonian AM73 & Spanish & -0.1023 & -0.0253 & 0.0517 \\
\hline Pericú BC30 & East Euro HG & -0.1030 & -0.0400 & 0.0230 \\
\hline Patagonian AM73 & Georgia HG & -0.1037 & -0.0287 & 0.0463 \\
\hline Pericú BC27 & Pre-LGM Euro & -0.1039 & 0.0291 & 0.1621 \\
\hline
\end{tabular}




\begin{tabular}{|c|c|c|c|c|}
\hline Target population & Source population 2 & $-1 \mathrm{SE}$ & Mean & $+1 \mathrm{SE}$ \\
\hline Patagonian AM72 & Tamil & -0.1059 & 0.1171 & 0.3401 \\
\hline Pericú BC27 & Iran EN & -0.1060 & 0.0110 & 0.1280 \\
\hline Patagonian AM71 & Georgia HG & -0.1064 & -0.0664 & -0.0264 \\
\hline Patagonian AM71 & Spanish & -0.1072 & -0.0682 & -0.0292 \\
\hline Pericú BC23 & West Euro HG & -0.1076 & 0.0534 & 0.2144 \\
\hline British Columbia 939 & West Euro HG & -0.1083 & -0.0783 & -0.0483 \\
\hline Pericú BC27 & West Euro HG & -0.1086 & 0.0214 & 0.1514 \\
\hline Pericú BC27 & Pit Grave CA & -0.1096 & 0.0304 & 0.1704 \\
\hline Patagonian AM73 & East Euro HG & -0.1097 & 0.0043 & 0.1183 \\
\hline Patagonian AM73 & Pit Grave CA & -0.1099 & -0.0199 & 0.0701 \\
\hline British Columbia 939 & Pre-LGM Euro & -0.1116 & -0.0846 & -0.0576 \\
\hline Mex mummy MOM6 & Mala & -0.1120 & 0.0360 & 0.1840 \\
\hline Patagonian AM71 & Pre-LGM Euro & -0.1129 & -0.0729 & -0.0329 \\
\hline British Columbia 939 & Pit Grave CA & -0.1152 & -0.0832 & -0.0512 \\
\hline Patagonian AM73 & Pre-LGM Euro & -0.1152 & -0.0372 & 0.0408 \\
\hline British Columbia 939 & Tamil & -0.1164 & -0.0874 & -0.0584 \\
\hline Patagonian AM71 & Tamil & -0.1170 & -0.0770 & -0.0370 \\
\hline Pericú BC27 & Tamil & -0.1186 & 0.0064 & 0.1314 \\
\hline Patagonian AM72 & Pre-LGM Euro & -0.1189 & 0.0861 & 0.2911 \\
\hline Patagonian AM71 & Mala & -0.1191 & -0.0801 & -0.0411 \\
\hline Patagonian AM73 & Mala & -0.1197 & -0.0417 & 0.0363 \\
\hline British Columbia 939 & Mala & -0.1216 & -0.0916 & -0.0616 \\
\hline Pericú BC27 & Mala & -0.1221 & -0.0031 & 0.1159 \\
\hline Patagonian AM73 & Tamil & -0.1228 & -0.0428 & 0.0372 \\
\hline Patagonian AM71 & West Euro HG & -0.1231 & -0.0801 & -0.0371 \\
\hline Patagonian AM73 & West Euro HG & -0.1241 & -0.0371 & 0.0499 \\
\hline Pericú BC23 & Mala & -0.1251 & 0.0379 & 0.2009 \\
\hline British Columbia 939 & Natufian & -0.1271 & -0.0971 & -0.0671 \\
\hline Pericú BC23 & Pit Grave CA & -0.1280 & 0.0460 & 0.2200 \\
\hline Patagonian AM72 & Pit Grave CA & -0.1299 & 0.1241 & 0.3781 \\
\hline Patagonian AM71 & Pit Grave CA & -0.1308 & -0.0828 & -0.0348 \\
\hline Patagonian AM71 & East Euro HG & -0.1344 & -0.0774 & -0.0204 \\
\hline
\end{tabular}




\begin{tabular}{|c|c|c|c|c|}
\hline Target population & Source population 2 & $-1 \mathrm{SE}$ & Mean & $+1 \mathrm{SE}$ \\
\hline British Columbia 939 & East Euro HG & -0.1434 & -0.1044 & -0.0654 \\
\hline Patagonian AM72 & West Euro HG & -0.1447 & 0.1213 & 0.3873 \\
\hline Mex mummy MOM6 & East Euro HG & -0.1657 & 0.0323 & 0.2303 \\
\hline Pericú BC27 & East Euro HG & -0.1731 & 0.0249 & 0.2229 \\
\hline Fuegian MA572 & Georgia HG & -0.1806 & -0.0816 & 0.0174 \\
\hline Fuegian MA572 & NW Asia Minor EN & -0.1941 & -0.0861 & 0.0219 \\
\hline Pericú BC23 & East Euro HG & -0.1991 & 0.0389 & 0.2769 \\
\hline Fuegian MA572 & Iran EN & -0.2024 & -0.0924 & 0.0176 \\
\hline Fuegian MA572 & West Euro HG & -0.2039 & -0.0859 & 0.0321 \\
\hline Patagonian AM72 & Natufian & -0.2101 & 0.2569 & 0.7239 \\
\hline Fuegian MA572 & Pre-LGM Euro & -0.2137 & -0.0997 & 0.0143 \\
\hline Fuegian MA572 & Spanish & -0.2184 & -0.1014 & 0.0156 \\
\hline Fuegian MA572 & Mala & -0.2217 & -0.1097 & 0.0023 \\
\hline Fuegian MA572 & Tamil & -0.2228 & -0.1018 & 0.0192 \\
\hline Fuegian MA572 & East Euro HG & -0.2326 & -0.0956 & 0.0414 \\
\hline Pericú BC28 & Natufian & -0.2424 & -0.1064 & 0.0296 \\
\hline Fuegian MA572 & Pit Grave CA & -0.2532 & -0.1122 & 0.0288 \\
\hline Fuegian MA572 & Natufian & -0.2882 & -0.1412 & 0.0058 \\
\hline Fuegian MA575 & Pre-LGM Euro & -0.3196 & -0.1466 & 0.0264 \\
\hline Fuegian MA575 & Iran EN & -0.3350 & -0.1380 & 0.0590 \\
\hline Fuegian MA575 & Georgia HG & -0.3576 & -0.1676 & 0.0224 \\
\hline Fuegian MA575 & Spanish & -0.3629 & -0.1779 & 0.0071 \\
\hline Fuegian MA575 & NW Asia Minor EN & -0.3717 & -0.1837 & 0.0043 \\
\hline Fuegian MA575 & Mala & -0.3774 & -0.1454 & 0.0866 \\
\hline Patagonian AM72 & East Euro HG & -0.3854 & 0.0356 & 0.4566 \\
\hline Fuegian MA575 & Natufian & -0.3864 & -0.1784 & 0.0296 \\
\hline Fuegian MA575 & Tamil & -0.3923 & -0.1723 & 0.0477 \\
\hline Fuegian MA575 & Pit Grave CA & -0.4451 & -0.2371 & -0.0291 \\
\hline Fuegian MA575 & West Euro HG & -0.4688 & -0.2238 & 0.0212 \\
\hline Fuegian MA575 & East Euro HG & -0.5707 & -0.2947 & -0.0187 \\
\hline
\end{tabular}


Figure 1: PCA of Amerindians, Europeans, and Middle Easterners

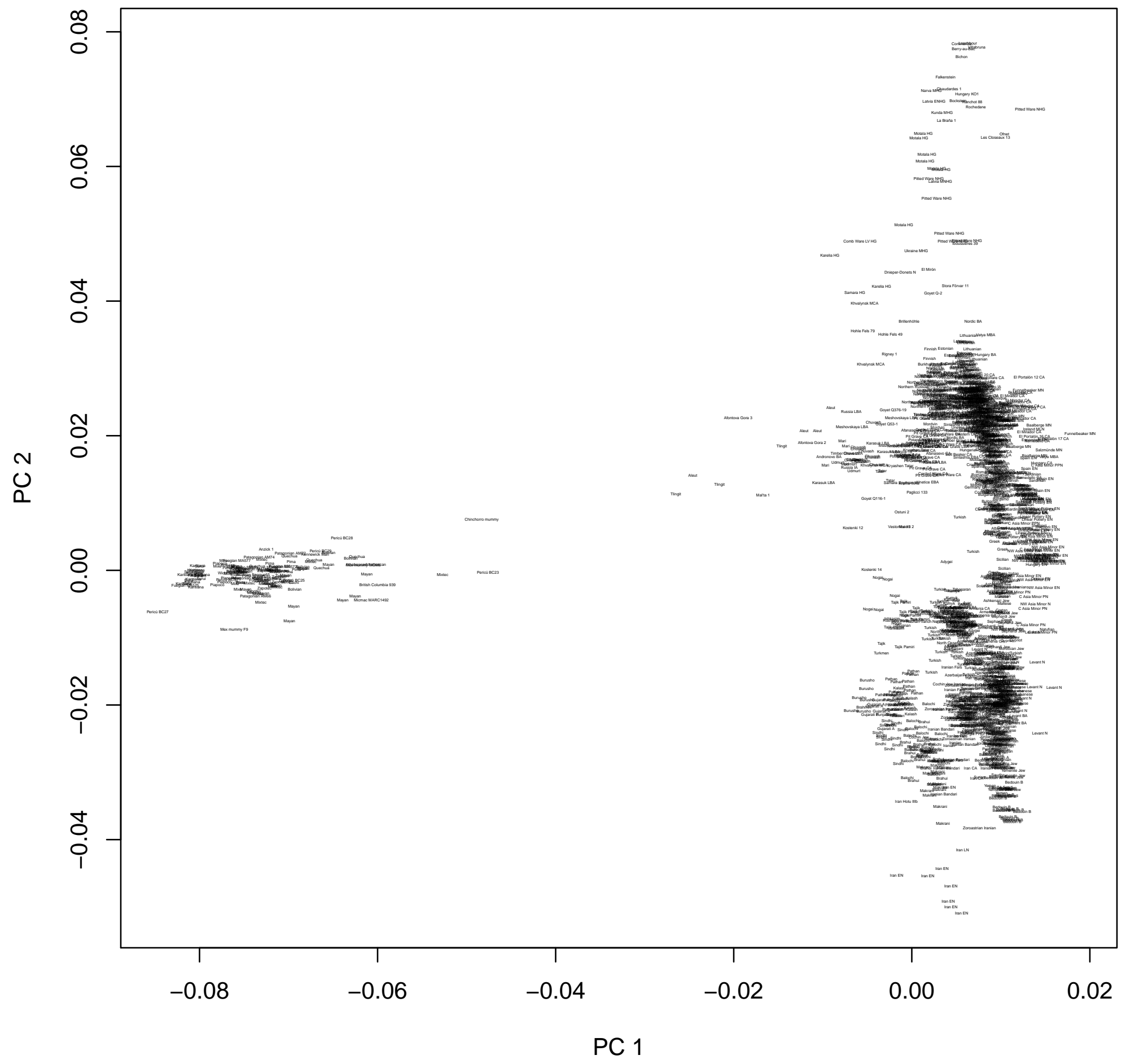


Figure 2: PCA of Amerindians and Europeans

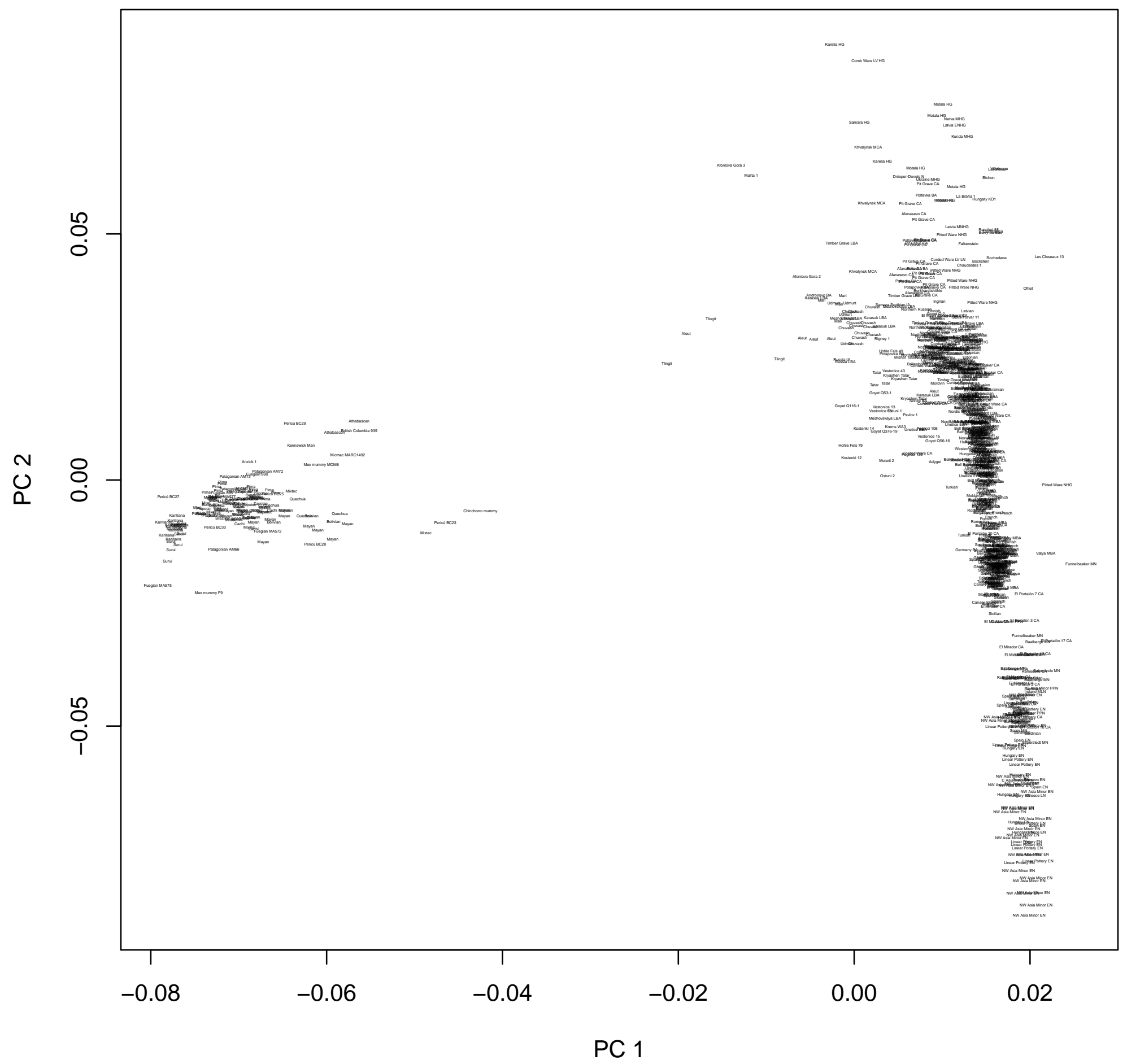







1 